\title{
Flebite em crianças e adolescentes que utilizaram cateter venoso periférico
}

RESUMO | Objetivo: Identificar a prevalência de flebite relacionada ao uso de cateter venoso periférico em crianças e adolescentes. Método: Estudo retrospectivo, quantitativo, com dados provenientes de notificação de flebite relacionada à terapia intravenosa periférica, no período de janeiro de 2012 a dezembro de 2016 em um hospital infantil na cidade de São Paulo. Resultados: A prevalência de flebite foi de 0,62\%, sendo 116 casos notificados em 18.924 crianças e adolescentes. Identificou-se associação significativa do grau de flebite e às variáveis: "Tipo de infusão" $(p=0,001)$ "Fármaco de alta osmolaridade" $(p=0,046)$ e "Uso de soro com eletrólitos" $(p=0,005)$. Conclusão: É fundamental que o enfermeiro avalie o tipo de terapêutica medicamentosa, rede venosa e clínica do paciente, indicando o dispositivo apropriado para essa finalidade, bem como, implementar indicadores de prevalência dos eventos adversos para prevenção e possíveis intervenções precoces, visando a segurança e eficácia na qualidade do cuidado de enfermagem.

Palavras-chaves: Cateteres; Criança; Enfermagem Pediátrica; Flebite; Prevalência.

ABSTRACT | Objective: To identify the prevalence of phlebitis related to the use of peripheral venous catheters in children and adolescents. Method: Retrospective, quantitative study, with data from notification of phlebitis related to peripheral intravenous therapy, from January 2012 to December 2016 in a children's hospital in the city of São Paulo. Results: The prevalence of phlebitis was $0.62 \%$, with 116 cases reported in 18,924 children and adolescents. A significant association was identified between the degree of phlebitis and the variables: "Type of infusion" ( $p=0.001)$ "High osmolarity drug" $(p=0.046)$ and "Use of serum with electrolytes" ( $p=0.005)$. Conclusion: It is essential that nurses evaluate the type of drug therapy, venous network and patient's clinic, indicating the appropriate device for this purpose, as well as implementing indicators of prevalence of adverse events for prevention and possible early interventions, aiming at safety and efficacy. in the quality of nursing care.

Keywords: Catheters; Child; Pediatric Nursing; Phlebitis; Prevalence.

RESUMEN | Objetivo: identificar la prevalencia de flebitis relacionada con el uso de catéteres venosos periféricos en niños y adolescentes. Método: estudio cuantitativo retrospectivo, con datos de notificación de flebitis relacionada con la terapia intravenosa periférica, de enero de 2012 a diciembre de 2016 en un hospital infantil de la ciudad de São Paulo. Resultados: La prevalencia de flebitis fue de $0.62 \%$, con 116 casos reportados en 18,924 niños y adolescentes. Se identificó una asociación significativa entre el grado de flebitis y las variables: "Tipo de infusión" ( $p=0.001)$ "Medicamento de alta osmolaridad" ( $p$ $=0.046)$ y "Uso de suero con electrolitos" $(p=0.005)$. Conclusión: es esencial que las enfermeras evalúen el tipo de terapia farmacológica, la red venosa y la clínica del paciente, indicando el dispositivo apropiado para este propósito, e implementando indicadores de prevalencia de eventos adversos para la prevención y posibles intervenciones tempranas, con el objetivo de seguridad y eficacia. en la calidad de la atención de enfermería.

Descriptores: Catéteres; Niño; Enfermería pediátrica; Flebitis; Predominio.

\section{Wilza Cabral Rodrigues da Silva}

Enfermeira. Mestre em Ciências da Saúde pelo Programa de Pós-Graduação em Ciências da Saúde do Instituto de Assistência Médica ao servidor publico Estadual (IAMSPE). Membro Coordenodor do Time de acesso vascular e terapia infusional do Hospital Infantil Cândido Fontoura (HICF).São Paulo/SP- Brasil

\section{Jaques Waisberg}

Médico. Pós-doutorado e Livre Docência pela Disciplina de Gastroenterologia Cirúrgica do Departamento de Cirurgia da EPM-UNIFESP. Coordenador do Programa de Pós-Graduação Stricto Sensu em Ciências da Saúde do Instituto de Assistência Médica ao Servidor Público Estadual (IAMSPE), Pró-Reitor de PósGraduação do IAMSPE, orientador colaborador e coordenador local do Programa de PósGraduação Stricto Sensu em Ciências da Saúde da FMABC. São Paulo/SP- Brasil.

\section{Gizelda Monteiro da Silva \\ Enfermeira. Doutora em Educação. Docente e orientadora do Programa de Mestrado em Ciências da Saúde do Instituto de Assistência Médica ao Servidor Público Estadual (IAMSPE). Membro da Câmara Técnica de Educação do Conselho Regional de Enfermagem de São Paulo. São Paulo/SP- Brasil.}

Recebido em: 13/04/2020

Aprovado em: 14/04/2020

\section{INTRODUÇÃO}

o ambiente hospitalar, o uso
de cateter venoso periférico
(CVP) é frequente em crianças hospitalizadas para a administração de fármacos, fluidos, componentes sanguíneos e nutricionais ${ }^{(1,2)}$.

Trata- se de um procedimento invasivo e complexo, pois envolve a abordagem a crianças, familiares e, faz-se necessário competência técnica, habilidade, conhecimentos de anatomia e fisiologia do sistema vascular e farmocoterapêutica ${ }^{(1,3,4)}$. Tornando-se um desafio em razão das características específicas dessa população, os critérios na escolha do CVP são determinados pela: idade da criança; qualidade e calibre do dispositivo; sua finalida- 
de; tempo de utilização; osmolaridade e $\mathrm{pH}$ dos fármacos. Outros fatores indispensáveis são as características do cateter e a escolha do curativo podem proporcionar conforto e segurança durante o tratamento ${ }^{(3,5-7)}$.

Cabe ressaltar que eventos adversos são complicações indesejadas, decorrentes do cuidado prestado, sendo evitáveis ${ }^{(5,7)}$.

A flebite caracteriza-se pela inflamação aguda na parede do vaso sanguíneo. Os sinais e sintomas são: edema, dor, desconforto e eritema ao redor do local de inserção do cateter vascular, sendo uma das complicações mais comuns da terapia intraveno$\mathrm{sa}^{(4,5,8)}$. A classificação da flebite pode ocorrer da seguinte forma: mecânica, química, bacteriana ou infecciosa e pós-infusional(5,8).

Com destaque, observa-se a importância do cumprimento das recomendações da Aliança Mundial para a Segurança do Paciente em prevenir a ocorrência de agravos à saúde dos pacientes $^{(9)}$.

Para maior conscientização e sensibilização junto à equipe de enfermagem sobre a segurança do paciente ${ }^{(5)} \mathrm{e}$ a criação de protocolo referente ao uso do CVP na terapêutica medicamentosa, promoverá uma gestão de qualidade. Nesse prisma, a implementação das práticas baseadas em evidências (PBE) reduz os fatores de risco relacionados à flebite ${ }^{(10)}$.

No tocante às escalas para avaliação e acompanhamento no grau de flebite, são ferramentas usadas em diversos serviços hospitalares com objetivo de estabelecer conduta pela enfermei$\mathrm{ra}^{(1,5,11)}$. A Escala de Maddox ${ }^{(11)}$ foi a primeira evidenciar a severidade de sinais clínicos desse evento adverso.

Nesse sentido, a temática é relevante na pratica diária da equipe de enfermagem por ser desafiadora a busca pela qualidade e segurança ao paciente no planejamento assistencial e, a redução dos eventos adversos con-

tribuem para uma terapêutica medicamentosa segura.

À luz da literatura apresentada, propomos responder à seguinte questão norteadora: Qual a prevalência de flebite em crianças e adolescentes que fizeram uso de cateter venoso periférico (CVP)? Este estudo tem como objetivo identificar a prevalência de flebite em crianças e adolescentes que fizeram uso de CVP, utilizando a escala de Maddox para classificação do grau de flebite.

\section{METODOLOGIA}

sobre a segurança do paciente ${ }^{(5)}$

e a criação de protocolo referente ao uso do CVP na terapêutica medicamentosa, promoverá

uma gestão de qualidade.

Nesse prisma, a implementação das práticas baseadas em evidências (PBE) reduz os fatores de risco relacionados à flebite $^{(10)}$.
Trata-se de um estudo de caso exploratório-descritivo, retrospectivo e documental, com elucidações quantitativas. Ocorreu em um hospital público pediátrico localizado na região metropolitana da Zona Leste de São Paulo, com atendimento a pacientes pediátricos na faixa etária entre 0 e 18 anos, exclusivo pelo Sistema Único de Saúde (SUS). Os dados foram levantados através das fichas de notificações de flebite preenchidas pela Enfermagem. A coleta de dados ocorreu entre outubro de 2014 e janeiro 2017, sendo realizada pela pesquisadora, após autorização da Diretoria de Divisão de Enfermagem.

Incluiram-se todas as fichas de notificação de flebite e seus respectivos graus em crianças e adolescentes que utilizaram CVP, de janeiro 2012 a dezembro de 2016. Não houve critérios de exclusão.

Quanto à avaliação do sítio de inserção do CVP, foi realizada pelos enfermeiros da instituição através da escala de Maddox para classificar o grau de flebite. A mesma, foi adaptada pelo serviço da seguinte forma: Grau 0: Ausência de reação; Grau 1: Sensibilidade ao toque sobre a porção I.V da cânula; Grau 2: Dor contínua, sem eritema; Grau 3: Dor contínua, com eritema e edema, veia dura palpável a menos de $8 \mathrm{~cm}$ acima do local I.V. (cânula); Grau 4: Dor contínua, com 
eritema e edema, endurecimento, veia endurecida palpável a mais de $8 \mathrm{~cm}$ do local I.V.; Grau 5: Trombose venosa aparente. Todos os sinais de 4+, mais fluxo venoso $=0$, pode ter sido interrompido devido à trombose $\mathrm{e}^{(11,12)}$.

A prevalência de flebite foi calculada pela seguinte fórmula: número de casos existentes de flebite/ número de pacientes com acesso periférico x $100^{(5)}$.

Para associação de variáveis qualitativas e o grau de flebite, são apresentadas tabelas com frequências cruzadas, assim como o valor $\mathrm{p}$ para o teste exato de Fisher. Foi considerado nível de significância de 5\%, ou seja, quando o valor p é inferior à 0,05 , temos evidência de existência de associação.

O estudo atendeu às questões éticas, de acordo com a Resolução do Conselho Nacional de Saúde (CNS) n. ${ }^{\circ}$ 466, de 12 de dezembro de 2012, aprovado no local de estudo e pelo Comitê de Ética em Pesquisa do Instituto de Assistência Médica do Servidor Público Estadual, sob o protocolo n. ${ }^{\circ}$ 2.057.166

Tabela 1. Distribuição segundo a frequências absolutas e percentuais para variáveis qualitativas significativa de acordo com o grau de flebite e resultado do teste exato de Fisher. São Paulo, SP, Brasil, 2017.

\begin{tabular}{lrrrrrrrrr} 
Grau de Flebite & \multicolumn{2}{c}{$\mathbf{1}-\mathbf{2}$} & & 3 & & $\mathbf{4}-\mathbf{5}$ & P-valor \\
& N & $\%$ & N & $\%$ & N & $\%$ & \\
& Tipo de infusão & & & & & \\
\hline Continua & 1 & $14,3 \%$ & 1 & $14,3 \%$ & 5 & $71,4 \%$ & 0,001 \\
\hline Continua e intermitente & 11 & $31,4 \%$ & 17 & $48,6 \%$ & 7 & $20,0 \%$ & \\
\hline Intermitente & 25 & $35,2 \%$ & 41 & $57,7 \%$ & 5 & $7,0 \%$ & \\
\hline Não informado* & 1 & $33,3 \%$ & 1 & $33,3 \%$ & 1 & $33,3 \%$ &
\end{tabular}

Fármaco alta osmolaridade

\begin{tabular}{lrrrrrrr} 
Sim & 2 & $33,3 \%$ & 1 & $16,7 \%$ & 3 & $50,0 \%$ & 0,046 \\
\hline Não & 36 & $32,7 \%$ & 59 & $53,6 \%$ & 15 & $13,6 \%$ & \\
\multicolumn{1}{c}{ Uso de soro com eletrólitos } & & & \\
\hline Nim & 10 & $25,0 \%$ & 18 & $45,0 \%$ & 12 & $30,0 \%$ & 0,005 \\
\hline Não informado* & 27 & $36,5 \%$ & 42 & $56,8 \%$ & 5 & $6,8 \%$ & \\
\hline
\end{tabular}

Nota: *As frequências "Não informado" não foram considerados no teste de Fisher.
Não foi identificado indício significativo associado aos graus de flebite às seguintes variaveis: gênero, local inserção cateter, calibre do cateter, curativo/ fixação, antibiotico, fármaco vesicante, fármaco irritante, fármaco extremo de $\mathrm{pH}$, associação de dois antibióticos, associação acima de dois fármacos.

Na Tabela 1, apresentamos os dados estatisticos referentes à associação significativa de flebite e às variáveis: "Tipo de infusão" ( $p=0,001)$ "Fármaco de alta osmolaridade" $(p=0,046)$ e "Uso de soro com eletrólitos" ( $p=0,005)$.

\section{DISCUSSÃO}

A Infusion Nurses Society (INS) estabelece uma prevalência de flebite de até $5 \%$ em crianças ou adultos ${ }^{(5)}$.

Ao se analisar as fichas de notificação de flebite, além de recurso aos prontuários, percebeu-se a falta de registro de alguns dados.Cabe ressaltar que a baixa prevalência de flebite sugere subnotificação. Tal fato pode ser razão da dificuldade de o enfermeiro identificar o evento adverso como prejudicial ao paciente; a baixa adesão do cumprimento dos protocolos na rotina de trabalho ou ainda a insegurança pela cultura de punição existente nas instituições, prevalecendo a omissão dos fatos. Pensando nesta problemática, o enfermeiro exerce um papel primordial no processo de gerenciamento da intervenção e manutenção da terapia intravenosa (TIV). Portanto, em relação ao registro, quando realizado de forma adequada, poderá ter impacto direto na redução dos casos de evento adverso relacionada à flebite. Neste contexto, é necessário que os registros sejam organizados e completos em relação aos aspectos da TIV. Assim, é preciso identificar possíveis fatores de risco para o surgimento de evento adverso, conforme preconizado pela Resolução n. ${ }^{\circ} 514$ do Conselho Federal de Enfermagem - COFEN ${ }^{(1,13)}$.

Infere-se ainda que o fato da baixa 
prevalência dos casos de flebite deve-se à utilização do Sistema de Distribuição de Medicamento por Dose Unitária (SDMDU) pelo hospital estudado, implementado desde 1995. O SDMDU é definido pela American Society Hospital of Pharmacists (ASPH) como "uma quantidade ordenada de medicamentos com formas e dosagens prontas para serem administradas a um paciente, de acordo com o prescrito para um determinado tempo" ${ }^{\prime(14)}$

No SDMDU, a dose do medicamento é embalada, identificada e dispensada pronta para ser administrada sem necessidade de transferências, cálculos e manipulação prévia por parte da enfermagem $^{(15)}$.

Este método incrementa a qualidade da assistência, reduzindo possíveis erros no preparo, diluição e administração dos fármacos, uma vez que a Farmácia Hospitalar fraciona os medicamentos utilizando a concentração máxima e o volume mínimo - famacocinética e farmadinâmica ${ }^{(14)}$.

Além disso, é preconizado perfusor (bomba seringa) para controle do tempo na administração dos fármacos nas Unidades de Terapia Intensiva Pediátrica e Neonatal e em crianças menores de um ano. Nas demais faixa etárias, a câmera graduada respeita o tempo de infusão de cada fármaco recomendado pelo fabricante. Pensando no serviço local, a Enfermagem procura implementar tais critérios, corroborando com as recomendações da ANVISA $^{(6)}$

Entretanto, a infusão intermitente pode ocasionar maior ocorrência de flebite, em razão da maior manipulação do cateter ${ }^{(16)}$.

Corroborando com estudo realizado em crianças, revela-se que fármacos com extremos de $\mathrm{pH}$, osmolaridade e infusão de eletrólitos associados a outros fármacos constituem um fator de risco aumentado ao desenvolvimento de flebite ${ }^{(1)}$.

Cabe enfatizar que fármacos ou soluções com osmolaridade acima de
Para prevenir a aparição de evento adverso de flebite, a ANVISA, a INS e as Diretrizes para Prevenção de Infecções Associadas a Cateteres Intravasculares (CDC) recomendam avaliação rotineira e frequente das condições do paciente, sítio de inserção, integridade da pele e do vaso, duração e tipo de terapia prescrita, integridade e permeabilidade do dispositivo, cobertura e estabilização estéril.
900mOsml/L também podem provocar alterações fisiológicas no endotélio venoso, devendo ser administrados em acesso venoso central(5).

Para minimizar os riscos de flebite, é fundamental que a equipe de enfermagem conheça o $\mathrm{pH}$, a osmolaridade, volume e diluente adequado, bem como o tempo de infusão dos fármacos ${ }^{(1,17)}$.

Em relação à flebite grau alto 4 ou 5, onde grau 4 apresenta dor contínua, com eritema e edema, endurecimento, veia endurecida palpável a mais de $8 \mathrm{~cm}$ do local I.V e; no Grau 5 apresenta trombose venosa aparente, todos os sinais de grau 4, mais fluxo venoso $=0$, tendo sido interrompidos pela trombose ${ }^{(11,12)}$.

Portanto, evidencia-se um problema aos profissionais de enfermagem no hospital pediátrico, pois os pacientes foram expostos à flebite. Assim, é essencial a implementação de estratégias para prevenção e identificação precoce dos sinais desta complicação(5).

Para prevenir a aparição de evento adverso de flebite, a ANVISA, a INS e as Diretrizes para Prevenção de Infecções Associadas a Cateteres Intravasculares (CDC) recomendam avaliação rotineira e frequente das condições do paciente, sítio de inserção, integridade da pele e do vaso, duração e tipo de terapia prescrita, integridade e permeabilidade do dispositivo, cobertura e estabilização estéril. Sugere-se também o empoderamento do paciente e/ou familiares nos cuidados, com o objetivo de identificar previamente os primeiros sinais e sintomas de flebite e complicações relacionadas à terapia intravenosa ${ }^{(5,6,18)}$.

A identificação precoce da dor e a remoção do cateter por esse motivo poderão diminuir a evolução do processo inflamatório para manifestações clínicas de maior comprometimento tecidual, bem como o grau mais grave de flebite ${ }^{(19)}$.

É imperiosa a avaliação dos riscos e benefícios de cada tipo de cateter, bem como considerar as preferências 
do paciente.Tal análise poderá considerar a indicação de outros cateteres venosos ao paciente, como o cateter venoso central de inserção periférica - PICC ${ }^{(17)}$. Como limitações do estudo, observou-se a falta de dados nas notificações sobre a patologia de base. Além disso, os registros das notificações eram realizados no surgimento da flebite, não havendo acompanhamento diário da punção venosa periférica, impossibilitando o comparativo da prevalência entre pacientes com e sem flebite.

\section{CONCLUSÃO}

Este estudo nos mostra indício significativo associado de flebite grau alto (4 ou 5) nas seguintes variaveis: "Tipo de infusão" ; "Fármaco de alta osmolaridade" e "Uso de soro com eletrólitos" em crianças e adolescentes durante o uso de Cateter Venoso Periferico. Desse modo, é fundamental que o enfermeiro avalie o tipo de terapêutica medicamentosa, rede venosa e clínica do paciente, indicando o dispositivo apropriado para essa finalidade, bem como, implementar indicadores de prevalência dos eventos adversos para prevenção e possíveis intervenções precoces, visando a segurança e eficácia na qualidade do cuidado de enfermagem.

* Parte da Dissertação apresentada ao Instituto de Assistência Médica ao Servidor Público Estadual de São Paulo, para obtenção do Título de Mestre em Ciências da Saúde pelo Programa de Pós-Graduação em Ciências da Saúde.

Caracterização de flebites em crianças que utilizaram cateteres venosos: incidência, possíveis riscos e assistência de Enfermagem. São Paulo / SP 2017

\section{Referências}

1. Bitencourt ES, Leal CN, Boostel R, Mazza VA, Felix JVC, Pedrolo E.prevalence of phlebitis related to the use of periphera intravenous devices in children.Cogitare Enferm [Internet]. 2018; (23)1:e4936. DOI: http://dx.doi.org/10.5380/ce.v23i1.49361.

2. Almeida TJC, Miranda JOF, Santos LM, Santana RCB, Camargo CL, Sobrinho CLN.

Peripheral venous accesses in hospitalized children: a photographic study. Rev enferm UFPE on-line [Internet]. 2016; 10(Supl. 2):701-7. DOI: 10.5205/reuol.6884-59404-2-SM-1.1002sup2016041.

3. Silva WCR, Oliboni MWCR, Borrel JG. Punção venosa periférica e central na criança: cuidados de enfermagem. In: BorrelJG,BarrosL, Lens SAF, Costa SAF, editoras. Administração de Medicamentos em Pediatria. São Caetano do Sul-SP: Ed.Yendis; 2016.p. 135-162.

4. Urbanetto JS, Peixoto CG, May TA. Incidence of phlebitis associated with the use of peripheral IV catheter and following catheter removal. Rev. Latino-Am Enfermagem [Internet]. 2016; 24:e2746. DOI: http:// dx.doi.org/10.1590/1518-8345.0604.2746.

5. Infusion Nurses Society (BR). Diretrizes práticas para terapia infusional. São Paulo; 2018.127p.

6. Ministério da Saúde, Agência Nacional de Vigilância Sanitária (BR). Medidas de prevenção de infecção relacionadas à assistência à saúde [Internet]. Brasilia (DF): MS,2017 [acesso em 10 jan 2020]. Disponível em: http://portal.anvisa.gov.br/documents/33852/3507912/ Caderno+4+-+Medidas+de+Preven $\% \mathrm{C} 3 \% \mathrm{~A} 7 \% \mathrm{C} 3 \% \mathrm{~A} 30+$ de+Infec $\%$ C3\%A7\%C3\%A30+Relacionada+\%C3\%A0+Assist\%C3\%AAncia+\%C3\%A0+Sa\%C3\%BAde/a3f23dfb-2c54-4e64-881 c-fccf9220c373.

7. Conselho Regional de Enfermagem de São Paulo (BR). Parecer Coren-SPCat n. ${ }^{\circ}$ 20/2010. Da fundamentação e análise. A terapia intravenosa (TIV) integra o cotidiano a enfermagem no tratamento dos agravos à saúde [Internet]. São Paulo (SP): Coren-SP, 2010 [acesso em 11 jan 2020]. Disponível em: http://www.corensp.gov.br/sites/default/ files/parecer_coren_sp_2010_20.pdf.

8. Urbanetto JS, Muniz FOM, Silva RM, Freitas APC, Oliveira APR, Santos JCR. Incidência de flebite e flebite pós $\neg$-infusional em adultos hospitalizados. Rev Gaúcha Enferm [Internet]. 2017; 38(2):e58793. DOI: http://dx.doi.org/10.1590/1983- 1447.2017.02.58793.

9. World Health Organization. Patient Safety: Making health care safer [Internet]. Geneva: WHO; 2017 [acesso em 10 jan 2020]. Disponível em: https://apps.who.int WHO-HIS-SDS-2017.11-eng.pdf.

10. Pimenta CAM, Francisco AA, Lopes CT, Nishi FA, Maia FOM, Shimoda GT, et al. Guia para a implementação de protocolos assistenciais de enfermagem: integrando protocolos, prática baseada em evidência e classificações de enfermagem [Internet]. São Paulo (SP): COREN-SP,
2017 [cesso em 13 jan 2020]. Disponivel em: http://www.corensp.gov. br/sites/default/files/guia_implementacao_protocolos_assistenciais enfermagemintegrando protocolos pratica baseada_em evidencia classificacao_enfermagem.pdf.

11. Maddox RR, Rush DR, Rapp RP, Foster TS, Mazella V, MckeanHE, et al. Double-blind study to investigat methods to prevent cephaloothin-induced phlebitis. American Journal of Hospital Pharmacy.1977; 34(1):29-34.

12. Araújo SAN. Acompanhamento de punção venosa periférica e central e escala demonitoramento de flebite "Maddox". 2010. Diretoria de Divisão de Enfermagem do Hospital Infantil Cândido Fontoura. São Paulo, 2010.

13. Conselho Federal de Enfermagem (BR). Resolução COFEN n. ${ }^{\circ} 514$, de 5 de maio de 2016. Guia de Recomendações para os registros de enfermagem no prontuário do paciente e outros documentos de enfermagem [Internet]. Brasília (DF): COFEN, 2016 [acesso em 10 jan 2020]. Disponível em: http://http://www.cofen.gov.br/resolucao-cofen-no-05142016_41295.html.

14. Araújo SAN. Sistema de distribuição de medicamentos por dose unitária(SDMDU). IN: Borre JG, Barros L, Lens SAF, Costa SAF. Administração de Medicamentos em Pediatria. São Caetano do Sul-SP: Yendis, 2016. 57-93.

15. Ministério da Saúde (BR). Protocolo de segurança na prescrição, uso e administração de medicamentos [Internet]. Brasília (DF): MS, 2013 [acesso em 13 jan 2020]. Disponível em: https://proqualis.net/ protocolo/protocolo-de-seguran $\% \mathrm{C} 3 \% \mathrm{~A} 7$ a-na-prescri $\% \mathrm{C} 3 \% \mathrm{~A} 7 \%$ C3\%A30-uso-e-administra\%C3\%A7\%C3\%A3o-de-medicamentos. 16. Enes SMS, Opitz SP, Faro ARMC, Pedreira MLG. Phlebitis associated with peripheral intravenous catheters in adults admitted to hospital in the Western Brazilian Amazon. Rev Esc Enferm USP [Internet]. 2016; 50(2):261-269. DOI: http://dx.doi.org/10.1590/S0080623420160000200012 .

17. Nobre ASP, Martins MDS. Prevalência de flebite da venopunção periférica: fatores associados. Rev. Enf. Ref. [Internet]. 2018; IV(16):127138. DOI: http://dx.doi.org/10.12707/RIV17058.

18. Guidelines for the Prevention of Intravascular Catheter-Related Infections, (CDC) [Internet]. 2011 [acesso em 11 jan 2020]. Disponível em:: htps://www.cdc.gov/hai/pdfs/bsi-guidelines-2011.pdf.

19. Braga LM, Parreira PM, Oliveira ASS, Mónico LSM, Arreguy-Sena $C$, Henriques MA. Phlebitis and infiltration: vascular trauma associated with the peripheral venous catheter. Rev. Latino-Am. Enfermagem [Internet]. 2018; 26:e3002. DOI: http://dx.doi.org/10.1590/15188345.2377.3002. 